Research Article

\title{
Static and Dynamic Characteristics of Composite Conoidal Shell Roofs
}

\author{
Kaustav Bakshi and Dipankar Chakravorty \\ Civil Engineering Department, Jadavpur University, Kolkata 700032, India \\ Correspondence should be addressed to Kaustav Bakshi; bakshi.kaustav@gmail.com
}

Received 30 April 2013; Revised 13 September 2013; Accepted 16 September 2013

Academic Editor: Rama Bhat

Copyright (c) $2013 \mathrm{~K}$. Bakshi and D. Chakravorty. This is an open access article distributed under the Creative Commons Attribution License, which permits unrestricted use, distribution, and reproduction in any medium, provided the original work is properly cited.

\begin{abstract}
A thorough review of the existing literature reflects that forced vibration studies of laminated composite conoidal shells with complicated boundary conditions are missing. Hence, the present paper aims to fill the lacuna. A finite element code utilizing eight-noded doubly curved elements together with modified Sanders' first approximation theory for thin shells is used to study the forced vibration behavior of moderately thin laminated composite conoidal shells subjected to three different uniformly distributed time-dependent forces. Newmark's direct time integration method is used to solve the dynamic problem. Results obtained using the present code are compared with the values available in the literature, and a good agreement of the results confirms the accuracy of the proposed code. The transient responses of the laminated shell are studied meticulously for parametric variations like boundary conditions and stacking orders of cross and angle-ply laminates and are compared with bending responses of the shell to conclude on the necessity of the dynamic study.
\end{abstract}

\section{Introduction}

Laminated composites gained popularity to fabricate plates and shells since second half of the last century. High strength/stiffness to weight ratio, low cost of fabrication, and better durability of the laminated composites popularized them in the weight-sensitive engineering applications. Moreover, the stiffness parameters of this material can be altered by varying the fiber orientations and lamina stacking sequences which made them a lucrative option to the engineers. Naturally, a good number of research reports started publishing on laminated plates and shells. A number of researchers like Reddy [1], Reddy and Chandrashekhara [2], Ribeiro [3], and Nanda and Bandyopadhyay $[4,5]$ reported bending and dynamic responses of laminated shell configurations.

A conoidal shell is doubly curved, easy to cast, added stiff surface which is used in the civil engineering industry to cover large column free open spaces as one sees in airport terminal buildings, shopping malls, and in car parking lots. Moreover, these shell structures allow entry of daylight and natural air which makes them popular roofing units to medicinal plants and food processing units. In a number of situations like drop hammer condition in the industry, snow loading in low temperature areas, the hit by wind borne debris develops dynamic forces on these shells. To ensure an uninterrupted service life of the shell roofs, their relative static and dynamic performances are needed to be understood in detail.

A number of researchers worked on isotropic and laminated composite conoidal shells. Static bending responses of isotropic conoidal shells were reported by Ghosh and Bandyopadhyay [6, 7]. The authors used finite element method and reported deflections and stress resultants of the conoidal shells. Chakravorty et al. [8] studied fundamental frequencies of composite conoidal shells with cut-outs. They also reported forced vibration responses of laminated conoidal shells with point supported boundaries. Later, Nayak and Bandyopadhyay [9] studied forced vibration responses of stiffened isotropic conoidal shells. Apart from the transient characteristics, the authors [10] also carried out free vibration analysis of composite conoidal shells. Das and Chakravorty [11, 12] worked on bending and free vibration 


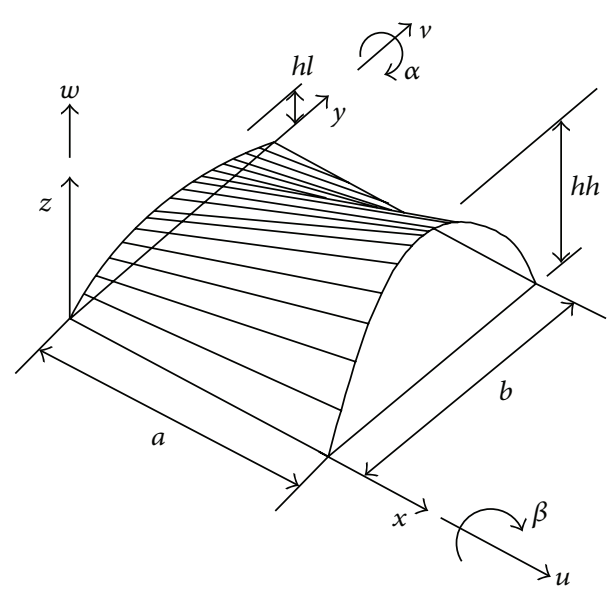

FIgURE 1: A typical conoidal shell.

of laminated conoidal shells with and without stiffeners, respectively. Bending characteristics of delaminated conoidal shells were reported by Kumari and Chakravorty $[13,14]$, while Pradyumna and Bandyopadhyay $[15,16]$, studied vibration and dynamic instability behaviors of laminated conoidal shells using the higher order shear deformation theory.

It is found from the literature survey that although a number of authors worked on dynamic analysis of laminated conoidal shells, none reported forced vibration responses of these shells with complicated boundary conditions. Hence, the present paper aims to study the deflections and stress resultants of laminated conoidal shells with complicated boundary conditions subjected to three different uniformly distributed time-dependent forces. Moreover, the static deflections and fundamental frequencies of these shells are also reported to understand the overall behavior of the conoidal shells.

\section{Mathematical Formulation}

2.1. Governing Differential Equation. Let us consider a laminated composite conoidal shell (Figure 1) of uniform thickness " $h$ " and radii of curvature " $R_{y y}$ " and " $R_{x y}$." Each of the thin laminae may be oriented at an angle " $\theta$ " with reference to the $x$-axis (Figure 3 ). Lagrange's equation of motion is derived using Hamilton's principle to express the governing differential equation of motion for an undamped forced vibration condition of the shell undergoing small displacements,

$$
\frac{d}{d t}\left\{\frac{\partial U_{2}}{\partial \dot{d}_{e}}\right\}-\left\{\frac{\partial U_{2}}{\partial d_{e}}\right\}+\left\{\frac{\partial}{\partial d_{e}}\left(U_{1}+U_{3}\right)\right\}=0,
$$

where $U_{1}$ : work done by conservative forces in a shell element and is given by

$$
U_{1}=\frac{1}{2} \int_{\bar{v}}\{\varepsilon\}^{T}\{\sigma\} d v
$$

$U_{2}$ : kinetic energy of the shell element and is given by

$$
U_{2}=\frac{1}{2} \iint_{A}\{\dot{d}\}^{T}[m][\dot{d}] d A,
$$

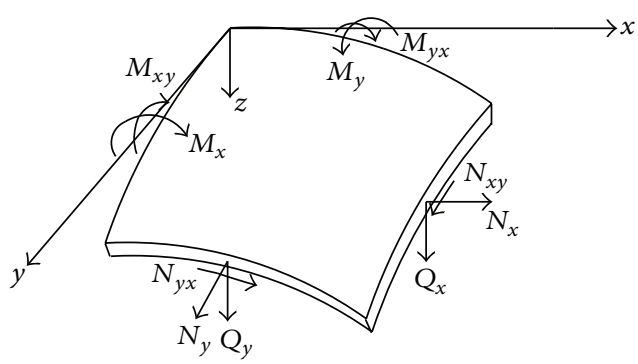

FIGURE 2: Shell stress resultants.

where $\{\dot{d}\}$ represents time derivative of shell deflections with respect to time.

$U_{3}$ : work done by external forces and is given by

$$
U_{3}=-\iint_{A}\{d\}^{T}\{q\} d A
$$

where $\{q\}$ indicates external transverse load intensity.

2.2. Laminate Constitutive Relationship. The laminate stress resultant vector $\{F\}$ is expressed using the following relationship:

$$
\{F\}=[D]\{\varepsilon\},
$$

where $[D]$ is the laminate constitutive relationship matrix and is adopted from Kumari and Chakravorty [13]. While obtaining the shear stress resultants, proper shear correction factors (as used by Das and Chakravorty [11]) are applied. The laminate stress resultant vector $\{F\}$ is expressed as

$$
\begin{aligned}
\{F\} & =\int_{-h / 2}^{+h / 2}\{\sigma\} d z \\
& =\int_{-h / 2}^{+h / 2}\left\{\begin{array}{llllllllll}
\sigma_{x} & \sigma_{y} & \tau_{x y} & \sigma_{x} \cdot z & \sigma_{y} \cdot z & \tau_{x y} \cdot z & \tau_{x z} & \tau_{y z}
\end{array}\right\}^{T} d z \\
& =\left\{\begin{array}{lllllllll}
N_{x} & N_{y} & N_{x y} & M_{x} & M_{y} & M_{x y} & Q_{x} & Q_{y}
\end{array}\right\}^{T}
\end{aligned}
$$

(see Figure 2).

2.3. Finite Element Formulation. An eight-noded curved quadratic isoparametric element (Figure 3 ) with $C^{0}$ continuity is used in the proposed finite element code. The element displacement field $\{d\}$ is described as follows:

$$
\{d\}=\left\{\begin{array}{llllll}
u & v & w & \alpha & \beta
\end{array}\right\}^{T},
$$

where $u, v$, and $w$ denote displacements of the shell along $x$, $y$, and $z$ axes and $\alpha$ and $\beta$ define rotations of the normal to the shell midsurface about $y$ and $x$ axes, respectively. The element degrees of freedom $\{d\}$ are expressed in terms of their nodal values $\left\{d_{e}\right\}$ by the following relationship:

$$
\{d\}=\sum_{i=1}^{8}\left[N_{i}\right]\left\{d_{i}\right\}
$$




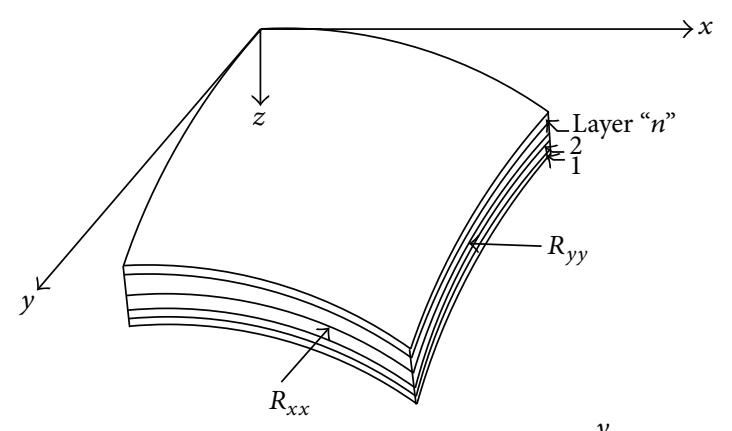

$R_{x x}$ is infinite for the present conoidal shell

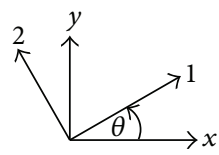

Fiber along 1-axis

Figure 3: The shell element.

where $\left[N_{i}\right]$ denotes the shape functions of the element and adopted is from Das and Chakravorty [11].

Following the modified Sanders' first approximation theory for thin shells, the midsurface strain vector of the shell is expressed as

$$
\begin{aligned}
& \left\{\varepsilon_{x}, \varepsilon_{y}, \gamma_{x y}, \gamma_{x z}, \gamma_{y z}\right\}^{T} \\
& \quad=\left\{\varepsilon_{x}^{0}, \varepsilon_{y}^{0}, \gamma_{x y}^{0}, \gamma_{x z}^{0}, \gamma_{y z}^{0}\right\}^{T}+z\left\{k_{x}, k_{y}, k_{x y}, k_{x z}, k_{y z}\right\}^{T},
\end{aligned}
$$

where

$$
\begin{gathered}
\left\{\begin{array}{c}
\varepsilon_{x}^{0} \\
\varepsilon_{y}^{0} \\
\gamma_{x y}^{0} \\
\gamma_{x z}^{0} \\
\gamma_{y z}^{0}
\end{array}\right\}=\left\{\begin{array}{c}
\frac{\partial u_{0}}{\partial x} \\
\frac{\partial v_{0}}{\partial y}-\frac{w}{R_{y y}} \\
\frac{\partial u_{0}}{\partial y}+\frac{\partial v_{0}}{\partial x}-\frac{2 w}{R_{x y}} \\
\alpha+\frac{\partial w}{\partial x} \\
\beta+\frac{\partial w}{\partial y}
\end{array}\right\}, \\
\left\{\begin{array}{c}
k_{x} \\
k_{y} \\
k_{x y} \\
k_{x z} \\
k_{y z}
\end{array}\right\}=\left\{\begin{array}{c}
\frac{\partial \alpha}{\partial x} \\
\frac{\partial \beta}{\partial y} \\
\frac{\partial \alpha}{\partial y}+\frac{\partial \beta}{\partial x} \\
0 \\
0
\end{array}\right\} .
\end{gathered}
$$

The strain vector is related to the nodal degrees of freedom by the strain displacement matrix $[B]$ which is the same used here as those were reported by Das and Chakravorty [11].

Using the strain displacement and laminate constitutive relations, (2) can be expressed as

$$
U_{1}=\frac{1}{2} \iint_{A}\left\{d_{e}\right\}^{T}[B]^{T}[D][B]\left\{d_{e}\right\} d x d y .
$$

Using (8), (3) is given by

$$
U_{2}=\frac{1}{2} \iint_{A}\left\{\dot{d}_{e}\right\}^{T}[N]^{T}[m][N]\left\{\dot{d}_{e}\right\} d x d y,
$$

where

$$
[m]=\left[\begin{array}{ccccc}
m & 0 & 0 & 0 & 0 \\
0 & m & 0 & 0 & 0 \\
0 & 0 & m & 0 & 0 \\
0 & 0 & 0 & I & 0 \\
0 & 0 & 0 & 0 & I
\end{array}\right] ; m=\rho h, \quad I=\frac{\rho h^{3}}{12} .
$$

Similarly, we get from (4),

$$
U_{3}=-\iint_{A}\left\{d_{e}\right\}^{T}[N]^{T}[q] d x d y .
$$

Using (11), (12), and (14), (1) can be expressed as

$$
\begin{aligned}
& \left\{\iint_{A}[N]^{T}[m][N] d x d y\right\}\left\{\ddot{d}_{e}\right\} \\
& \quad+\left\{\iint_{A}[B]^{T}[D][B] d x d y\right\}\left\{d_{e}\right\}=\iint_{A}[N]^{T}[q] d x d y .
\end{aligned}
$$

The coefficient of the element acceleration vector $\left\{\ddot{d}_{e}\right\}$ of (15) represents the element mass matrix $\left[M_{e}\right]$, and that of the element displacement vector $\left\{d_{e}\right\}$ represents the element stiffness matrix $\left[K_{e}\right]$. The term on the right-hand side of (15) represents the element load vector $\left\{Q_{e}\right\}$.

Thus, (15) results in

$$
\left[M_{e}\right]\left\{\ddot{d}_{e}\right\}+\left[K_{e}\right]\left\{d_{e}\right\}=\left\{Q_{e}\right\},
$$

where

$$
\begin{gathered}
{\left[M_{e}\right]=\iint_{A}[N]^{T}[m][N] d x d y,} \\
{\left[K_{e}\right]=\iint_{A}[B]^{T}[D][B] d x d y,} \\
\left\{Q_{e}\right\}=\iint_{A}[N]^{T}\{q\} d x d y .
\end{gathered}
$$

The element mass matrix, stiffness matrix, and load vector are transformed to isoparametric coordinates $\xi$ and $\eta$ for numerical integration by Gauss quadrature rule. The element matrices are assembled to get the respective global matrices with due consideration for curvature of the conoidal shell surface [17].

Consider

$$
[K]=\sum_{i=1}^{n e}\left[K_{e}\right], \quad[M]=\sum_{i=1}^{n e}\left[M_{e}\right], \quad[Q]=\sum_{i=1}^{n e}\left[Q_{e}\right] .
$$

The dynamic equation of motion in the global form is

$$
[M]\{\ddot{d}\}+[K]\{d\}=Q .
$$




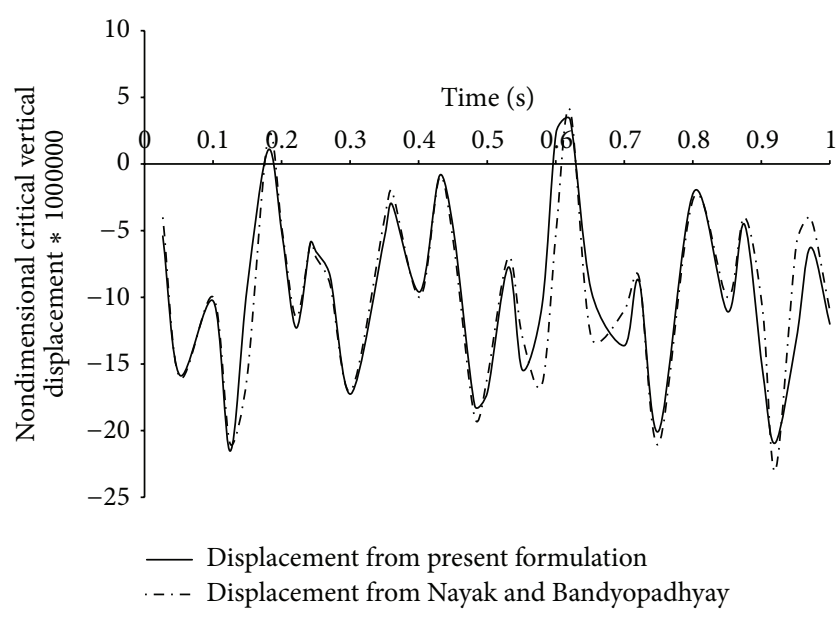

FIgURE 4: Dynamic response of isotropic conoidal shell under uniformly distributed step load. $a / b=1, b / h=250, b / h_{h}=0.15$, $h_{l} / h_{h}=0.25, b=25.0 \mathrm{~cm}, E=25.491 \times 10^{9} \mathrm{~N} / \mathrm{m}^{2}, v=0.15$, and $\rho=2500 \mathrm{~kg} / \mathrm{m}^{3}$.

TABLE 1: Nondimensional central displacements $\left(\bar{w} \times 10^{3}\right)$ of simply supported composite spherical shell under uniformly distributed load.

\begin{tabular}{lccc}
\hline Lamination & $0^{\circ} / 90^{\circ}$ & $0^{\circ} / 90^{\circ} / 0^{\circ}$ & $0^{\circ} / 90^{\circ} / 90^{\circ} / 0^{\circ}$ \\
\hline Reddy [1] & 16.980 & 6.697 & 6.833 \\
Present FEM $(2 \times 2)$ & 8.294 & 5.116 & 4.644 \\
$(4 \times 4)$ & 16.898 & 6.724 & 6.820 \\
$(6 \times 6)$ & 16.969 & 6.710 & 6.826 \\
$(8 \times 8)$ & 17.009 & 6.707 & 6.835 \\
\hline
\end{tabular}

$a / b=1, a / h=100, E_{11}=25 E_{22}, G_{12}=G_{13}=0.5 E_{22}, G_{23}=0.2 E_{22}, v=$ $0.25, E_{22}=10^{6} \mathrm{~N} / \mathrm{cm}^{2}, R / a=10^{30}$.

2.4. The Static Problem. If the inertia force term of (19) is dropped, and the displacement and load vectors are assumed to be time independent, then the following equation of static equilibrium is obtained:

$$
[K]\{d\}=\{Q\} .
$$

The previous equation is solved by the Gauss elimination method [18].

2.5. The Free Vibration Problem. If the load vector of (19) is dropped, the equation of free vibration is obtained as

$$
\begin{gathered}
{[K]\{d\}+[M]\{\ddot{d}\}=0,} \\
\text { or, }\left|[K]-\omega^{2}[M]\right|=0 .
\end{gathered}
$$

The free vibration analysis involves determination of natural frequencies and is solved by subspace iteration algorithm [18].

2.6. The Forced Vibration Problem. The global force vector in (19) is transient in nature and solved using Newmark's direct time integration method [18].
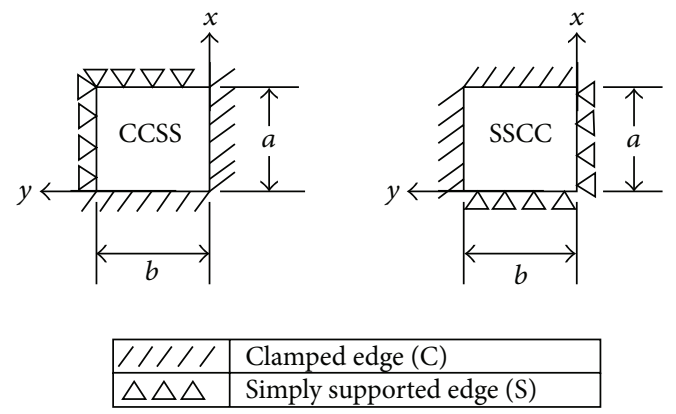

FIGURE 5: The boundary conditions.

TABLE 2: Nondimensional fundamental frequencies $(\varrho)$ of simply supported composite spherical shell.

\begin{tabular}{lccc}
\hline Lamination & $0^{\circ} / 90^{\circ}$ & $0^{\circ} / 90^{\circ} / 0^{\circ}$ & $0^{\circ} / 90^{\circ} / 90^{\circ} / 0^{\circ}$ \\
\hline Reddy [1] & 9.687 & 15.183 & 15.184 \\
Present FEM $(2 \times 2)$ & 14.897 & 15.209 & 15.221 \\
$(4 \times 4)$ & 9.722 & 15.209 & 15.222 \\
$(6 \times 6)$ & 9.691 & 15.179 & 15.195 \\
$(8 \times 8)$ & 9.681 & 15.180 & 15.183 \\
\hline
\end{tabular}

$a / b=1, a / h=100, E_{11}=25 E_{22}, G_{12}=G_{13}=0.5 E_{22}, G_{23}=0.2 E_{22}, v=$ $0.25, E_{22}=10^{6} \mathrm{~N} / \mathrm{cm}^{2}$, and $R / a=10^{30}$.

\section{Numerical Problems}

In order to verify the correctness of the static bending and free vibration formulations of the proposed code, the static deflections and fundamental frequencies of laminated spherical shell are evaluated and compared with the values reported by Reddy [1]. The comparisons are shown in Tables 1 and 2 with which the material and geometric properties of the shell are also reported as footnote. A comparison of results for varying mesh size are also reported in the tables.

The accuracy of the proposed code in predicting dynamic deflections of a conoidal shell is verified by comparing the present results with the values reported by Nayak and Bandyopadhyay [9]. The authors worked on isotropic conoidal shell, and the present code is modified accordingly by assigning equal values of elastic and shear moduli along all the directions. The comparison of results is furnished in Figure 4 where the material and geometric properties of the isotropic shell are also reported.

Apart from the verification problems, the present code is further utilized to study forced vibration responses of composite conoidal shells with two different complicated boundary conditions (Figure 5). The shells are subjected to three different time-dependent forces which are depicted in Figure 6. Keeping the total laminate thickness constant, symmetric and antisymmetric two, three, and four-layered laminates of cross and angle-ply laminations are considered. The nondimensional downward static deflections, nondimensional fundamental frequencies, and dynamic magnification of deflections for all three load cases of the conoidal shell are reported in Table 3. The nondimensional dynamic displacements are furnished in Table 4. The dynamic magnification factors 

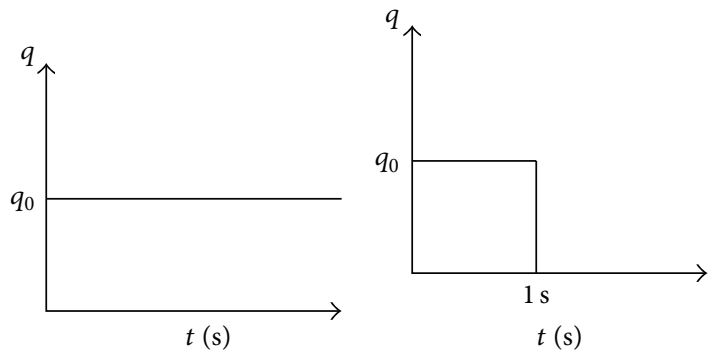

(a) A step load

(b) A rectangular pulse load

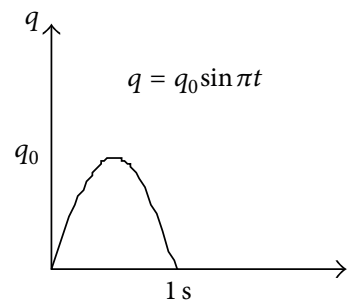

$t(\mathrm{~s})$

(c) Half sinusoidal pulse load

Figure 6: The time-dependent forces.

(hence forth referred as DMF) are calculated as the ratios of maximum dynamic responses to the corresponding static ones. The material and geometric properties of the conoidal shells are furnished with the tables. In case of bending analysis, the magnitude of the static load is considered equal to the peak step load value of load-time history considered here.

\section{Results and Discussion}

Static deflections and fundamental frequencies obtained using the present formulation are in close agreement [refer to Tables 1 and 2] with the values reported by Reddy [1]. Close agreement of static deflections as reported in Table 1 ensures accurate bending formulation for laminated shells in the current code. The deflection values monotonically increase as the finite element mesh is made finer in steps because a coarse mesh indicates a rigid modelling of the plate stiffness. For the $0^{0} / 90^{\circ}$ laminate, showing orthropy about the midplane, interestingly the deflection improves greatly when the mesh changes from a $2 \times 2$ to a $4 \times 4$ grid. For other laminates, the same trend is observed but the $2 \times 2$ mesh does not yield results which are so widely different from the converged ones. Correct incorporation of mass matrix of laminated shells is proved by the results of the second benchmark problem indicated in Table 2 . The frequency values decrease gradually as the mesh is made finer for the reasons explained previously. As it is found that a $2 \times 2$ mesh grossly overestimates the plate stiffness for $0^{0} / 90^{\circ}$ lamination, as a natural outcome the frequency value for this grid is much higher than the converged result. The response curves furnished in Figure 4 show that the natures of published response curves and those obtained by present approach are in close agreement and thus validates correct incorporation of Newmark's method in the present code.

The shells are ranked from 1 to 16 in terms of static and dynamic deflections with the first rank being given to the shell option showing the least value. In cases of fundamental frequency, the first rank goes to the shell exhibiting the highest value. Such ranks are provided in parentheses in Tables 3 and 4 and are very helpful to understand the relative behaviors of the shell combinations comprehensively.

The complicated boundary conditions considered here (Figure 5) have equal numbers of support degrees of freedom locked but arranged in a different manner. Naturally, a practicing engineer may be curious to know the relative performances of the conoidal shells in terms of these twoedge conditions. It is interesting to note from Table 3 that for any given lamination the CCSS boundary condition shows less static deflections when they are compared with the values obtained for the SSCC one. The conoidal shell is curved along one of its plan directions and has straight edges along the other plan direction. The arch direction of the shell is relatively stronger than the beam direction as it combines the bending and axial rigidity together to sustain external loads. But unlike the cylindrical shell, the conoidal shell has its curved boundaries at different elevations. This is why the bending stiffness of the shell is enhanced substantially on clamping the lower parabolic arch of the shell, and the static deflections decrease. The fundamental frequencies of the conoidal shell listed in the same table also vary in a similar manner as it was observed in case of the static deflections, although the symmetric angle-ply laminations show marginally higher values for the SSCC shell. The $45^{0} /-45^{\circ} / 45^{0}$ and $45^{\circ} /-45^{\circ} /-45^{0} / 45^{0}$ laminates yield $3.71 \%$ and $2.18 \%$ higher frequency values, respectively, for the SSCC boundary condition. This observation leads to conclude that clamping the lower arch of the conoid is advantageous to achieve higher bending and dynamic stiffnesses.

The dynamic deflections of all the shell options considered here are studied for three different load cases: step load of infinite duration (load case I), step load of finite duration (load case II), and half sinusoidal pulse load (dynamic load case III). The results are furnished in Table 4 . The results show that the dynamic deflections follow the same pattern as it was noted in cases of static deflections and fundamental frequencies where the CCSS shell performed better than the SSCC one. Although the magnification factors for all the load cases listed in Table 3 vary in a different manner where in a number of cases the SSCC shell shows lesser DMF values, it is found from the table that the DMF values for dynamic load cases I and II are all greater than 2. The load-time history of load cases I and II is identical up to 1 second, and after that the dynamic load corresponding to load case I continues to act with the same intensity, and for load case II, the load is withdrawn. Interestingly, the magnification factors are same for both the load cases or marginally higher for load case II. In load case III, both the application and withdrawn of pressure are gradual and this is why the DMF values are marginally greater than unity. 
TABLE 3: Nondimensional responses of the conoidal shell.

\begin{tabular}{|c|c|c|c|c|c|c|}
\hline \multirow{2}{*}{ Boundary condition } & \multirow{2}{*}{ Lamination (degree) } & \multirow{2}{*}{ Static deflections } & \multirow{2}{*}{ Fundamental frequency } & \multicolumn{3}{|c|}{ Dynamic magnification factors } \\
\hline & & & & Load case I & Load case II & Load case III \\
\hline \multirow{8}{*}{ CCSS } & $0 / 90$ & $0.000327(15)$ & $55.61(15)$ & $2.10(4)$ & $2.10(4)$ & $1.05(10)$ \\
\hline & $0 / 90 / 0$ & $0.000256(11)$ & $70.52(8)$ & $2.37(14)$ & $2.37(14)$ & $1.05(11)$ \\
\hline & $0 / 90 / 0 / 90$ & $0.000252(10)$ & $65.81(13)$ & $2.07(2)$ & $2.07(2)$ & $1.04(9)$ \\
\hline & 0/90/90/0 & $0.000243(9)$ & $71.37(7)$ & $2.24(11)$ & $2.24(11)$ & $1.05(12)$ \\
\hline & $45 /-45$ & $0.000104(3)$ & $70.06(9)$ & $2.18(8)$ & $2.18(8)$ & $1.01(1)$ \\
\hline & $45 /-45 / 45$ & $0.000116(4)$ & $82.46(6)$ & $2.08(3)$ & $2.08(3)$ & $1.02(5)$ \\
\hline & $45 /-45 / 45 /-45$ & $0.000083(1)$ & $89.15(1)$ & $2.15(5)$ & $2.15(5)$ & $1.01(2)$ \\
\hline & $45 /-45 /-45 / 45$ & $0.000091(2)$ & $86.90(4)$ & $2.16(7)$ & $2.16(7)$ & $1.02(6)$ \\
\hline \multirow{8}{*}{ SSCC } & $0 / 90$ & $0.000394(16)$ & $53.10(16)$ & $2.06(1)$ & $2.07(1)$ & $1.05(13)$ \\
\hline & $0 / 90 / 0$ & $0.000279(12)$ & $68.36(10)$ & $2.38(16)$ & $2.38(16)$ & $1.05(14)$ \\
\hline & $0 / 90 / 0 / 90$ & $0.000306(14)$ & $62.16(14)$ & $2.15(6)$ & $2.15(6)$ & $1.05(15)$ \\
\hline & $0 / 90 / 90 / 0$ & $0.000279(13)$ & $68.21(11)$ & $2.37(15)$ & $2.37(15)$ & $1.05(16)$ \\
\hline & $45 /-45$ & $0.000186(8)$ & $68.13(12)$ & $2.25(12)$ & $2.25(12)$ & $1.01(3)$ \\
\hline & $45 /-45 / 45$ & $0.000137(6)$ & $85.52(5)$ & $2.29(13)$ & $2.29(13)$ & $1.03(7)$ \\
\hline & $45 /-45 / 45 /-45$ & $0.000138(7)$ & $87.25(3)$ & $2.18(9)$ & $2.18(9)$ & $1.01(4)$ \\
\hline & $45 /-45 /-45 / 45$ & $0.000133(5)$ & $88.80(2)$ & $2.22(10)$ & $2.22(10)$ & $1.03(8)$ \\
\hline
\end{tabular}

$a / b=1, a / h=100, h_{h} / a=0.2, h_{l} / h_{h}=0.25, E_{11}=25 E_{22}, G_{12}=G_{13}=0.5 E_{22}, G_{23}=0.2 E_{22}, v_{12}=0.25$, and $\rho=100 \mathrm{Nsec}^{2} / \mathrm{m}^{4}$.

Values in parentheses indicate ranks for respective shell actions.

TABLE 4: Nondimensional dynamic deflections of the conoidal shell.

\begin{tabular}{|c|c|c|c|c|}
\hline \multirow{2}{*}{ Boundary condition } & \multirow{2}{*}{ Lamination (degree) } & \multicolumn{3}{|c|}{ Dynamic deflections } \\
\hline & & Load case I & Load case II & Load case III \\
\hline \multirow{8}{*}{ CCSS } & $0 / 90$ & $0.000687(15)$ & $0.000687(15)$ & $0.000342(15)$ \\
\hline & $0 / 90 / 0$ & $0.000598(11)$ & $0.000597(11)$ & $0.000265(11)$ \\
\hline & $0 / 90 / 0 / 90$ & $0.000522(9)$ & $0.000522(9)$ & $0.000262(10)$ \\
\hline & $0 / 90 / 90 / 0$ & $0.000546(10)$ & $0.000546(10)$ & $0.000256(9)$ \\
\hline & $45 /-45$ & $0.000227(3)$ & $0.000227(3)$ & $0.000105(3)$ \\
\hline & $45 /-45 / 45$ & $0.000241(4)$ & $0.000241(4)$ & $0.000118(4)$ \\
\hline & $45 /-45 / 45 /-45$ & $0.000178(1)$ & $0.000178(1)$ & $8.35 E-05(1)$ \\
\hline & $45 /-45 /-45 / 45$ & $0.000196(2)$ & $0.000196(2)$ & $9.24 E-05(2)$ \\
\hline \multirow{8}{*}{ SSCC } & $0 / 90$ & $0.000811(16)$ & $0.000814(16)$ & $0.000413(16)$ \\
\hline & $0 / 90 / 0$ & $0.000665(14)$ & $0.000665(14)$ & $0.000295(12)$ \\
\hline & $0 / 90 / 0 / 90$ & $0.000658(12)$ & $0.000658(12)$ & $0.000323(14)$ \\
\hline & 0/90/90/0 & $0.000662(13)$ & $0.000662(13)$ & $0.000295(13)$ \\
\hline & $45 /-45$ & $0.000418(8)$ & $0.000418(8)$ & $0.000188(8)$ \\
\hline & $45 /-45 / 45$ & $0.000315(7)$ & $0.000315(7)$ & $0.000142(7)$ \\
\hline & $45 /-45 / 45 /-45$ & $0.000301(6)$ & $0.000301(6)$ & $0.000139(6)$ \\
\hline & $45 /-45 /-45 / 45$ & $0.000294(5)$ & $0.000294(5)$ & $0.000136(5)$ \\
\hline
\end{tabular}

$a / b=1, a / h=100, h_{h} / a=0.2, h_{l} / h_{h}=0.25, E_{11}=25 E_{22}, G_{12}=G_{13}=0.5 E_{22}, G_{23}=0.2 E_{22}, v_{12}=0.25$, and $\rho=100 \mathrm{Nsec}^{2} / \mathrm{m}^{4}$.

Values in parentheses indicate ranks for respective shell actions.

It is also interesting to observe that for both the boundary conditions considered here the angle-ply shells yield lesser values of static and dynamic deflections when they are compared with their counterparts among cross-ply shells. It is further noted form Table 3 that among the symmetric and antisymmetric angle-ply laminates, the four-layered antisymmetric one $\left(45^{0} /-45^{0} / 45^{0} /-45^{0}\right)$ shows the best overall performance for the CCSS boundary condition. This means that in order to get the least possible static and dynamic deflections of a conoidal shell, an engineer's choice will be the CCSS boundary condition with the four-layered antisymmetric angle ply laminate $\left(45^{0} /-45^{0} / 45^{0} /-45^{0}\right)$. The above conclusion is further reinforced by the fact that in terms of fundamental frequency also this particular laminate 


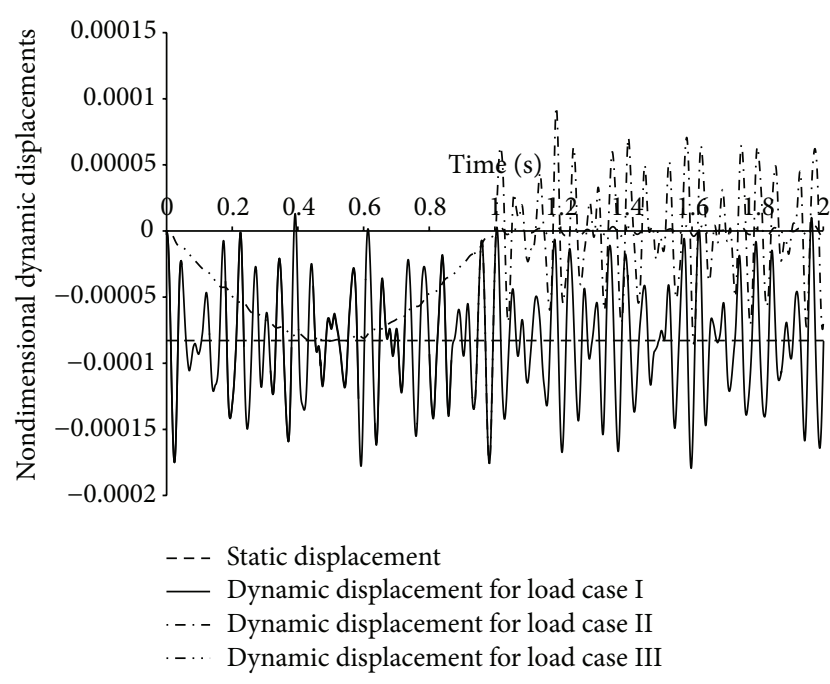

FIGURE 7: Nondimensional dynamic deflections $(\bar{w})$ of the conoidal shell at $x=0.25 \mathrm{~m}$ and $y=0.19 \mathrm{~m}$. Boundary condition: CCSS. Lamination: $45^{0} /-45^{0} / 45^{0} /-45^{0}$.

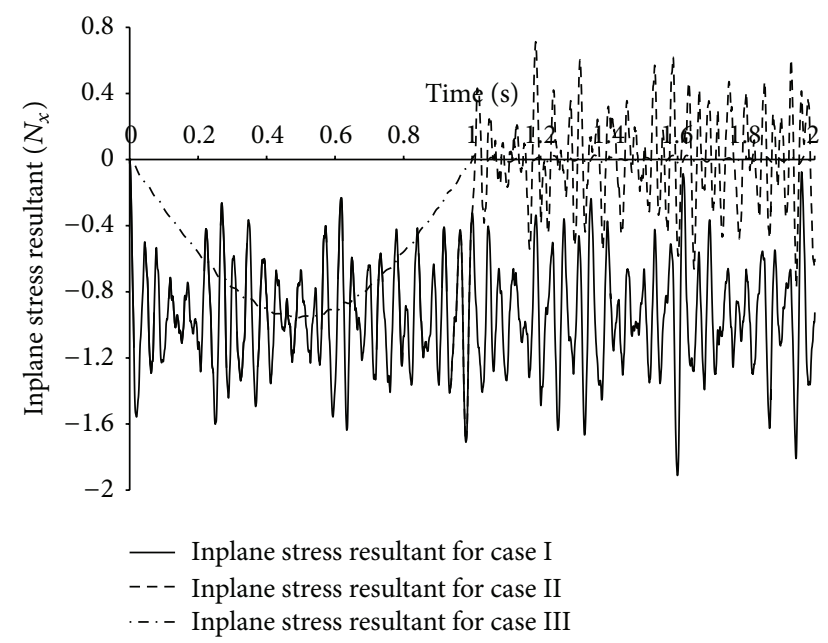

Figure 8: Nondimensional force resultants $\left(\bar{N}_{x}\right)$ of the conoidal shell at $x=0.25 \mathrm{~m}$ and $y=0.19 \mathrm{~m}$. Boundary condition: CCSS. Lamination: $45^{0} /-45^{0} / 45^{0} /-45^{0}$.

with the aforesaid boundary condition is the best available choice.

To study the transient responses of the composite conoidal shells after removal of the dynamic load, a more detailed transient analysis is carried out, and the results are furnished graphically in Figures 7, 8, 9, 10, and 11. The shell option showing the best overall performance is chosen for that study. The nondimensional dynamic deflections and force and moment resultants of the shell are studied. The shell responses are reported for all three load cases, and for each of them the study is carried out upto 2 seconds, that is, for a period of 1 second even after the load is withdrawn to see the after effect of the transient load. Dynamic responses of the shell are studied at the location of the maximum static displacement, and the respective $x$ and $y$ coordinates are

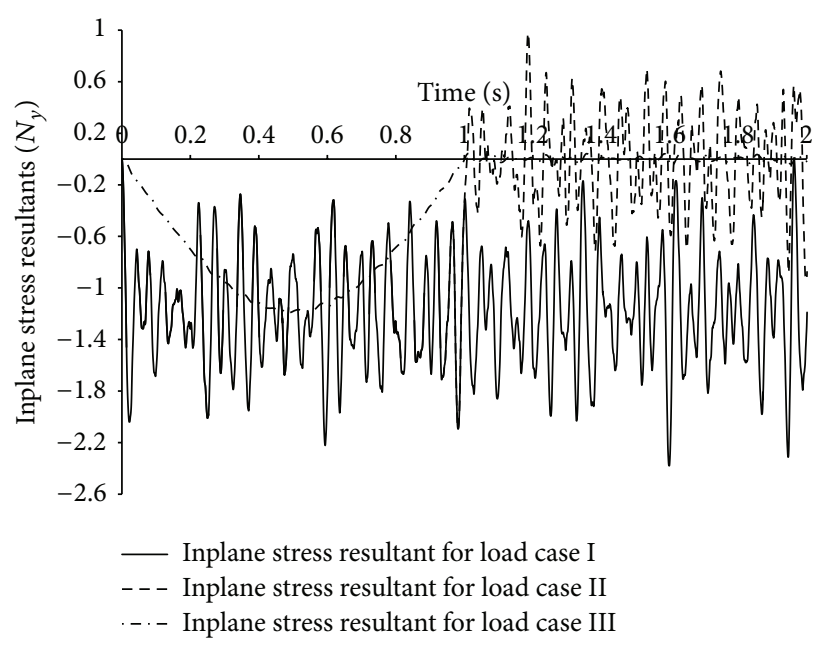

Figure 9: Nondimensional force resultants $\left(\bar{N}_{y}\right)$ of the conoidal shell at $x=0.25 \mathrm{~m}$ and $y=0.19 \mathrm{~m}$. Boundary condition: CCSS. Lamination: $45^{\circ} /-45^{0} / 45^{0} /-45^{0}$.

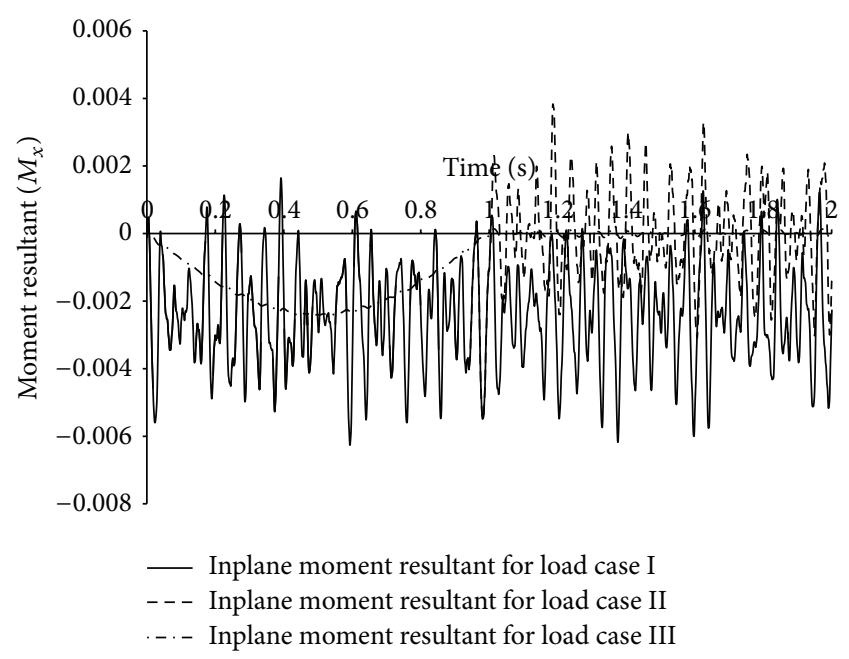

FIgURE 10: Nondimensional moment resultants $\left(\bar{M}_{x}\right)$ of the conoidal shell at $x=0.25 \mathrm{~m}$ and $y=0.19 \mathrm{~m}$. Boundary condition: CCSS. Lamination: $45^{0} /-45^{0} / 45^{0} /-45^{0}$.

presented with the figures. The loading conditions for load cases I and II are in fact identical up to time $=1$ second. This is why the response curves up to 1 second for these two load cases coincides. In both of these cases since the load is suddenly applied, the elastic rebound of the structure is quite strong, and sharp closely spaced crests and troughs are noted. For load case III, however, as the load is very gradually applied and withdrawn and load is of lesser magnitude, the deflection response curve almost resembles the shape of the loading curve.

The dynamic deflections for load cases I and II are reported along with the static deflection in Figure 7, and they are observed to oscillate around the static deflection upto 1 second. After the sudden removal of the dynamic load in case of load case II, the shell response magnifies drastically and even results in upward movements. This is 


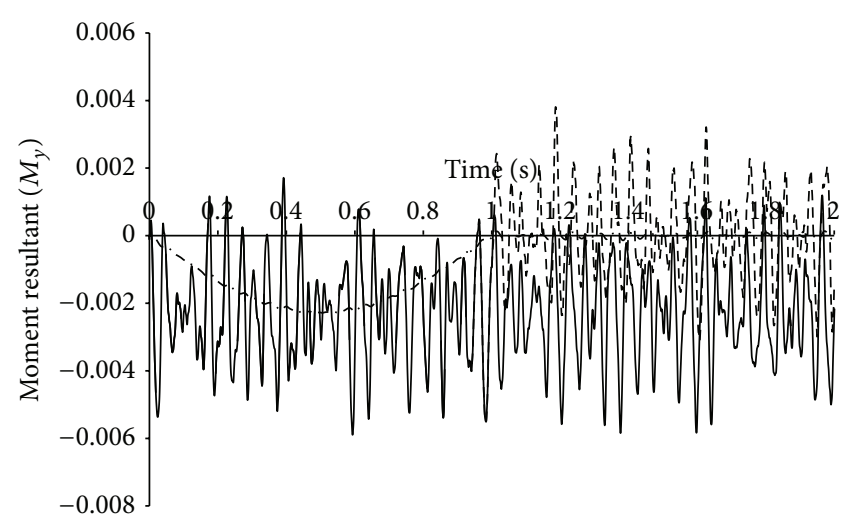

_ Inplane moment resultant for load case I

- - Inplane moment resultant for load case II

. - - Inplane moment resultant for load case III

FIGURE 11: Nondimensional moment resultants $\left(\bar{M}_{y}\right)$ of the conoidal shell at $x=0.25 \mathrm{~m}$ and $y=0.19 \mathrm{~m}$. Boundary condition: CCSS. Lamination: $45^{0} /-45^{0} / 45^{0} /-45^{0}$.

why the DMF values for load cases I and II are almost the same or marginally higher for load case II. This observation also brings out the importance of forced vibration analysis as static simplification of dynamic problems using a suitable factor cannot account for such reversal of dynamic displacements. It is also important to note here that in case of the dynamic loads, its time variation is more significant than the magnitude. The dynamic deflections for load case I continue its same vibrating pattern for 2 seconds. On the other hands for load case III when the load is applied and withdrawn gradually, the shell vibrates with positive and negative peaks of equal magnitude and in a frequency which is nearly equal to its fundamental frequency.

The force and moment resultants of the composite shell (Figures 8, 9, 10, and 11) follow the same pattern as it was observed in case of the dynamic deflections. Reversals of all the force and moment resultants are noted in the figures although it is more prominent for the moment resultants compared to than that of the force resultants in case of load case I where the dynamic load continued to act for 2 seconds.

\section{Conclusion}

The following conclusions can be drawn from this study.

(1) The present code accurately incorporates the finite element method to solve static, free, and forced vibration problems of laminated composite conoidal shells as it is indicated by the solutions of the benchmark problems.

(2) It is concluded that among the boundary conditions considered in the present study, the lower parabolic arch of the conoidal shell should be clamped to achieve higher bending and dynamic stiffnesses.

(3) It is further indicated that among the considered laminations, the $45^{0} /-45^{0} / 45^{0} /-45^{0}$ laminate shows the best overall performance by yielding the least static and dynamic deflections and the highest fundamental frequency.

(4) When the dynamic responses of the composite shell are studied for varying time, it is found that the deflections and stress resultants show reversal in nature. Hence a full dynamics analysis is needed to be carried out to get the magnitudes of the reversed stresses, imposing a factor of safety on the static values cannot take care of this phenomenon.

\section{Notations}

\begin{tabular}{|c|c|}
\hline A: & Area of the shell \\
\hline$a, b:$ & Length and width of shell in plan \\
\hline$D:$ & Flexural rigidity matrix of the shell \\
\hline$\{u\}:$ & Global displacement vector \\
\hline$\ddot{u}:$ & Global acceleration vector \\
\hline$E_{11}, E_{22}, E_{33}:$ & Young's moduli \\
\hline 1,2 and $3:$ & Local coordinates of a lamina \\
\hline$G_{12}, G_{13}, G_{23}:$ & Shear moduli \\
\hline$h h, h l:$ & $\begin{array}{l}\text { Higher and lower heights of the } \\
\text { conoidal shell, respectively }\end{array}$ \\
\hline$h:$ & Shell thickness \\
\hline$M_{x}, M_{y}, M_{x y}:$ & Moment resultants per unit length \\
\hline $\bar{M}_{x}, \bar{M}_{y}:$ & $\begin{array}{l}\text { Nondimensional moment } \\
\text { resultants }\left[=\left(M_{x} \text { or } M_{y}\right) / q a^{2}\right]\end{array}$ \\
\hline $\bar{M}_{x y}:$ & $\begin{array}{l}\text { Nondimensional torsion resultant } \\
{\left[=M_{x y} / q a b\right]}\end{array}$ \\
\hline$N_{x} N_{y} N_{x y}:$ & Force resultants per unit length \\
\hline $\bar{N}_{x}, \bar{N}_{y}:$ & $\begin{array}{l}\text { Nondimensional inplane force } \\
\text { resultants }\left[=\left(N_{x} \text { or } N_{y}\right) / q a\right]\end{array}$ \\
\hline $\bar{N}_{x y}:$ & $\begin{array}{l}\text { Nondimensional inplane shear resultant } \\
{\left[=N_{x y} / q a\right]}\end{array}$ \\
\hline ne: & Number of elements \\
\hline$Q_{x}, Q_{y}:$ & $\begin{array}{l}\text { Transverse shear resultants per unit } \\
\text { length }\end{array}$ \\
\hline$q_{0}:$ & Peak value of the transient loads \\
\hline$R_{x y}:$ & $\begin{array}{l}\text { Radius of cross curvature of conoidal } \\
\text { shell }\end{array}$ \\
\hline$R_{y y}:$ & $\begin{array}{l}\text { Radius of curvature of conoidal shell } \\
\text { along } y \text {-axis }\end{array}$ \\
\hline$R:$ & Radius of the spherical shell \\
\hline$u, v, w:$ & $\begin{array}{l}\text { Translational degree of freedoms along } \\
x-, y \text {-, and } z \text {-directions, respectively }\end{array}$ \\
\hline $\bar{v}:$ & Volume of the shell \\
\hline $\bar{w}:$ & $\begin{array}{l}\text { Nondimensional transverse } \\
\text { displacement }\left[=w E_{22} h^{3} /\left(q a^{4}\right)\right]\end{array}$ \\
\hline$x, y, z:$ & Global coordinates axes \\
\hline$\alpha, \beta$ & $\begin{array}{l}\text { Rotations of the shell about } y \text { - and } \\
x \text {-axes, respectively }\end{array}$ \\
\hline$\gamma_{x y}, \gamma_{x z}, \gamma_{y z}$ & Shear strains \\
\hline$\varepsilon_{x}, \varepsilon_{y}:$ & $\begin{array}{l}\text { Inplane strains along } x \text { - and } y \text {-axes, } \\
\text { respectively }\end{array}$ \\
\hline$\theta:$ & $\begin{array}{l}\text { Angle of lamination with respect to } \\
x \text {-axis of the conoidal shell }\end{array}$ \\
\hline$v_{i j}:$ & Poisson's ratio \\
\hline
\end{tabular}




$$
\begin{array}{ll}
\xi, \eta: & \text { Natural coordinates of isoparametric ele- } \\
& \text { ments } \\
\rho: & \text { Mass density } \\
\sigma_{x}, \sigma_{y}: & \begin{array}{l}
\text { Inplane stresses along } x \text { - and } y \text {-axes, res- } \\
\text { pectively }
\end{array} \\
\tau_{x y}, \tau_{x z}, \tau_{y z}: & \text { Shear stresses } \\
k_{x}, k_{y}, k_{x y}: & \text { Curvatures of conoidal shell due to load } \\
\omega: & \text { Fundamental frequency in radian/sec } \\
\bar{\omega}: & \text { Nondimensional fundamental frequency } \\
& {\left[=\omega a^{2}\left(\rho / E_{22} h^{2}\right)^{1 / 2}\right] .}
\end{array}
$$

\section{Acknowledgment}

The first author gratefully acknowledges the financial assistance of the Council of Scientific and Industrial Research, India, through the Senior Research Fellowship vide Grant no. 09/096 (0686) 2k11-EMR-I.

\section{References}

[1] J. N. Reddy, "Exact solutions of moderately thick laminated shells," Journal of Engineering Mechanics, vol. 110, no. 5, pp. 794$809,1984$.

[2] J. N. Reddy and K. Chandrashekhara, "Geometrically nonlinear transient analysis of laminated, doubly curved shells," International Journal of Non-Linear Mechanics, vol. 20, no. 2, pp. 79-80, 1985.

[3] P. Ribeiro, "Forced large amplitude periodic vibrations of cylindrical shallow shells," Finite Elements in Analysis and Design, vol. 44, no. 11, pp. 657-674, 2008.

[4] N. Nanda and J. N. Bandyopadhyay, "Nonlinear transient response of laminated composite shells," Journal of Engineering Mechanics, vol. 134, no. 11, pp. 983-990, 2008.

[5] N. Nanda and J. N. Bandyopadhyay, "Geometrically nonlinear transient analysis of laminated composite shells using the finite element method," Journal of Sound and Vibration, vol. 325, no. 1-2, pp. 174-185, 2009.

[6] B. Ghosh and J. N. Bandyopadhyay, "Bending analysis of conoidal shells using curved quadratic isoparametric element," Computers and Structures, vol. 33, no. 3, pp. 717-728, 1989.

[7] B. Ghosh and J. N. Bandyopadhyay, "Bending analysis of conoidal shells with cut-outs," Computers and Structures, vol. 53, no. 1, pp. 9-18, 1994.

[8] D. Chakravorty, P. K. Sinha, and J. N. Bandyopadhyay, "Applications of FEM on free and forced vibration of laminated shells," Journal of Engineering Mechanics, vol. 124, no. 1, pp. 1-8, 1998.

[9] A. N. Nayak and J. N. Bandyopadhyay, "Dynamic response analysis of stiffened conoidal shells," Journal of Sound and Vibration, vol. 291, no. 3-5, pp. 1288-1297, 2006.

[10] A. N. Nayak and J. N. Bandyopadhyay, "Free vibration analysis of laminated stiffened shells," Journal of Engineering Mechanics, vol. 131, no. 1, pp. 100-105, 2005.

[11] H. S. Das and D. Chakravorty, "Bending analysis of stiffened composite conoidal shell roofs through finite element application," Journal of Composite Materials, vol. 45, no. 5, pp. 525-542, 2011.

[12] H. S. Das and D. Chakravorty, "Natural frequencies and mode shapes of composite conoids with complicated boundary conditions," Journal of Reinforced Plastics and Composites, vol. 27, no. 13, pp. 1397-1415, 2008.
[13] S. Kumari and D. Chakravorty, "On the bending characteristics of damaged composite conoidal shells: a finite element approach," Journal of Reinforced Plastics and Composites, vol. 29, no. 21, pp. 3287-3296, 2010.

[14] S. Kumari and D. Chakravorty, "Bending of delaminated composite conoidal shells under uniformly distributed load," Journal of Engineering Mechanics, vol. 137, no. 10, pp. 660-668, 2011.

[15] S. Pradyumna and J. N. Bandyopadhyay, "Static and free vibration analyses of laminated shells using a higher-order theory," Journal of Reinforced Plastics and Composites, vol. 27, no. 2, pp. 167-186, 2008.

[16] S. Pradyumna and J. N. Bandyopadhyay, "Dynamic instability behavior of laminated hypar and conoid shells using a higherorder shear deformation theory," Thin-Walled Structures, vol. 49, no. 1, pp. 77-84, 2011.

[17] R. D. Cook, Finite Element Modeling for Stress Analysis, John Wiley and Sons, New York, NY, USA, 1995.

[18] K. J. Bathe, Finite Element Procedures, Prentice Hall, New Jersey, NJ, USA, 1996. 

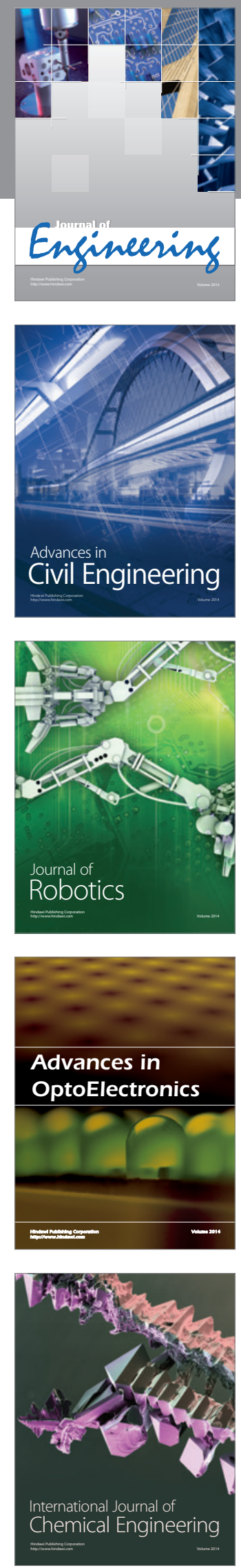

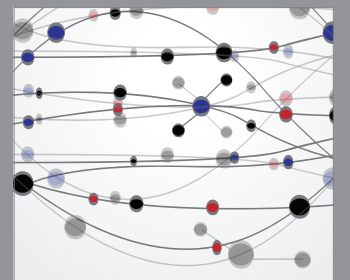

The Scientific World Journal
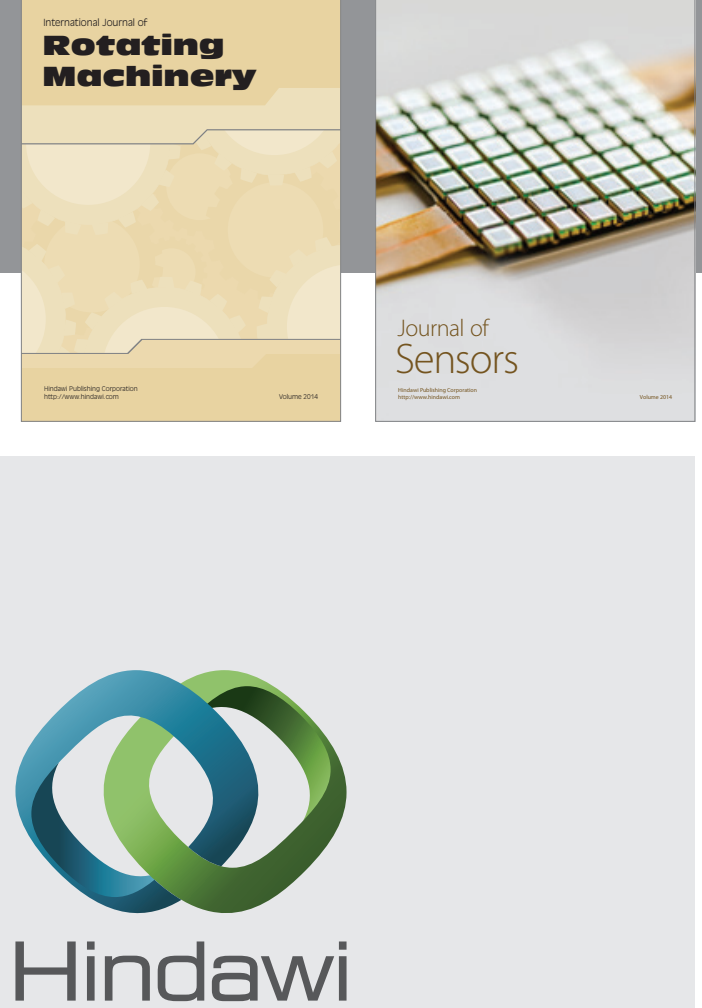

Submit your manuscripts at http://www.hindawi.com
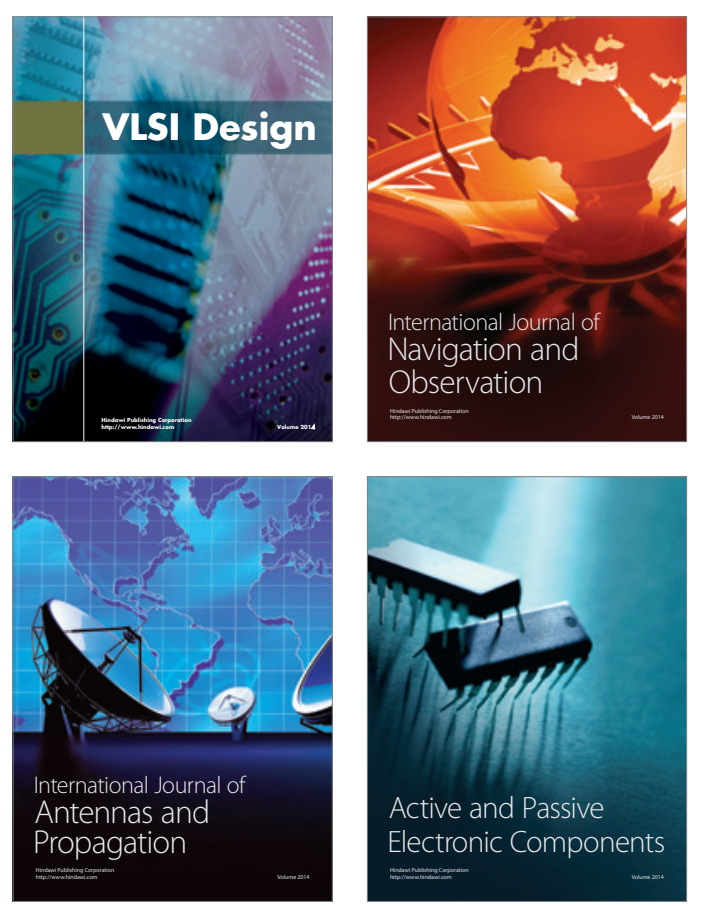
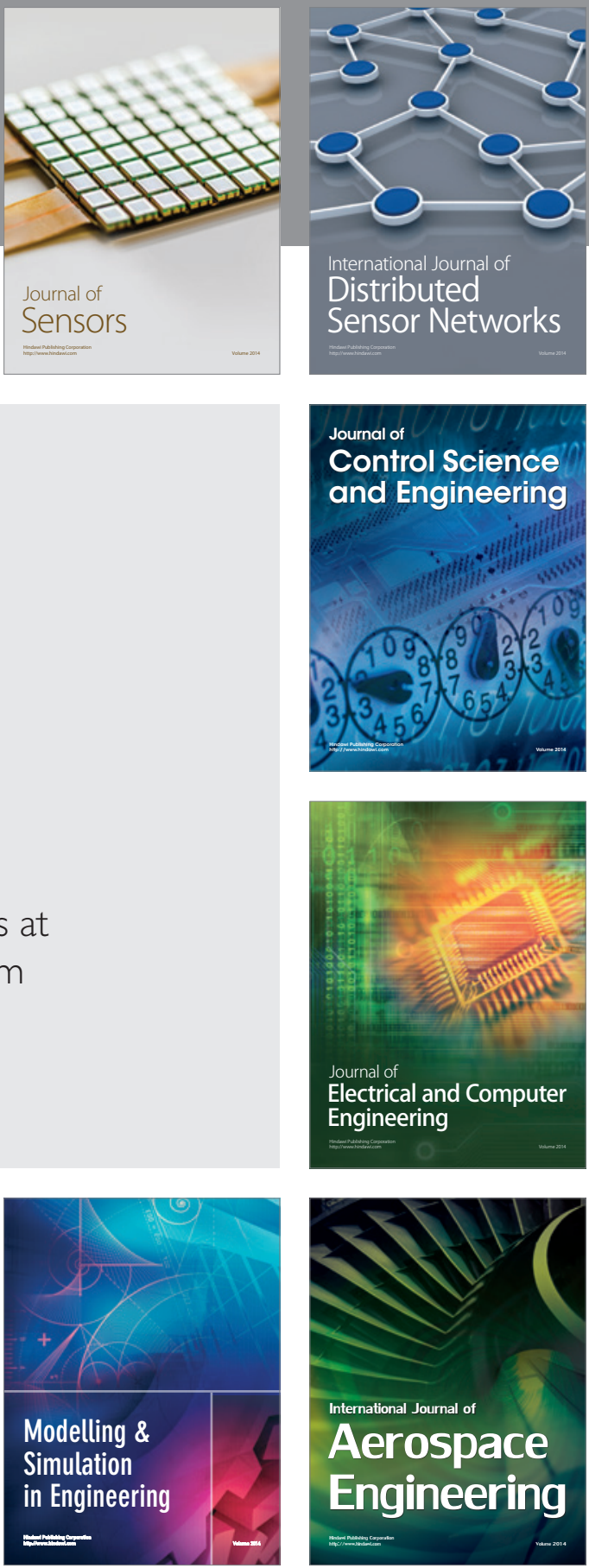

Journal of

Control Science

and Engineering
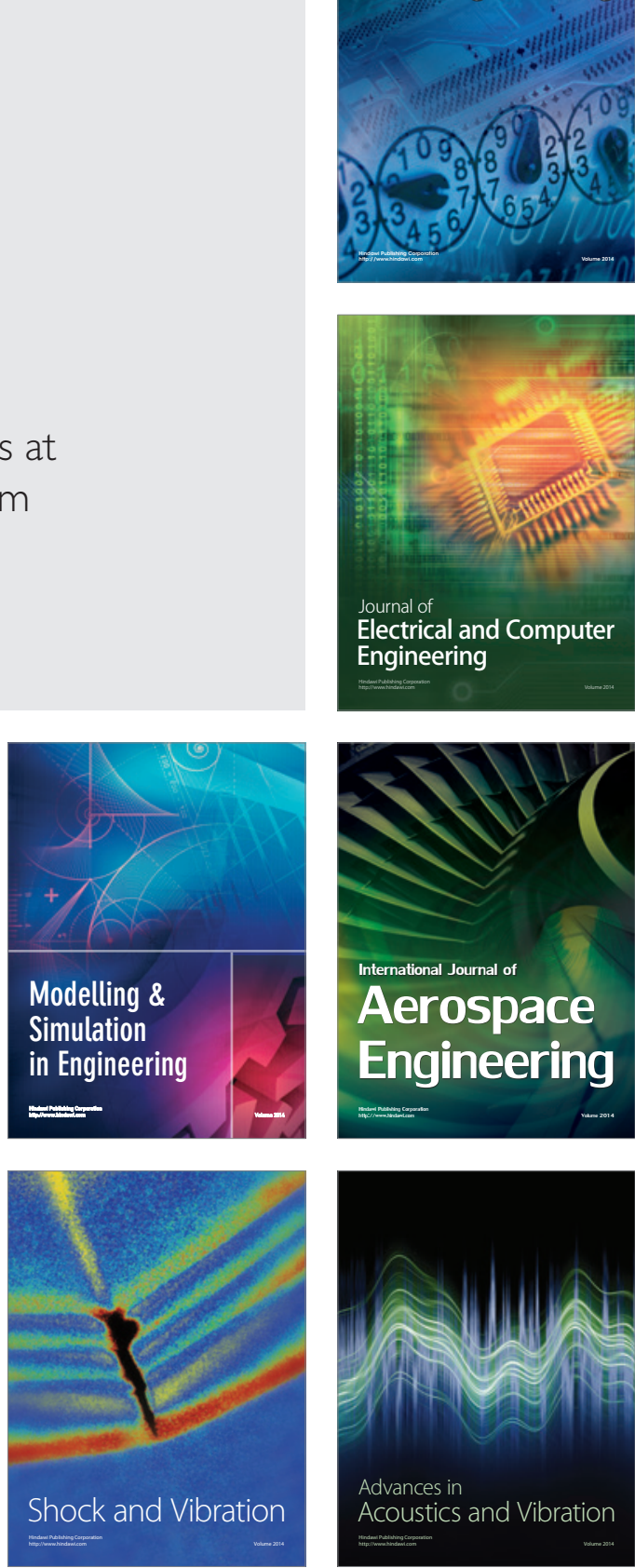